\title{
Osteoid Osteoma of the Thumb: A Case Report
}

\author{
Balazs Galdi • John T. Capo • Ali Nourbakhsh • \\ Francis Patterson
}

Received: 24 November 2009 / Accepted: 14 January 2010/Published online: 12 February 2010

(C) American Association for Hand Surgery 2010

\begin{abstract}
Osteoid osteoma is a rare occurrence in the hand, and only a small percentage affects the thumb and distal phalanges. An 18-year-old right-hand-dominant man presented to our office with an approximately 1-year history of left thumb pain without any history of trauma. He had seen several doctors previously and undergone multiple diagnostic tests with no definitive diagnosis. Plain radiographs and computed tomography at our institution were consistent with the diagnosis of osteoid osteoma. The patient was treated with surgical excision of the lesion without bone grafting. The diagnosis of osteoid osteoma was confirmed by pathology. At 6 months follow-up, the patient showed complete resolution of pain and full restoration of hand function. This case demonstrates that osteoid osteoma should not be forgotten as a differential diagnosis in patients with finger pain, especially in individuals who have not yet or just recently have reached skeletal maturity.
\end{abstract}

Keywords Osteoid osteoma P Phalanx · Tumor Resection Thumb

\section{Introduction}

Osteoid osteoma is a benign disease process of bone that usually affects children, adolescents, and young adults, with the majority of patients being between the ages of 10 and 25 years [8]. It is the third most common benign tumor, comprising $12 \%$ of benign tumors and $3 \%$ of all tumors, and has a maleto-female ratio of 2:1 [11]. Patients affected with this disease most typically complain of severe, unrelenting pain, worse at night and dramatically relieved by oral non-steroidal antiinflammatory medications (NSAIDs). Since osteoid osteoma

B. Galdi · J. T. Capo $(\bowtie) \cdot$ A. Nourbakhsh $\cdot$ F. Patterson Orthopedics Department, UMDNJ-New Jersey Medical School, 90 Bergen Street, DOC 1200,

Newark, NJ 07103, USA

e-mail: Capojt@umdnj.edu is a vascular tumor, substances that cause vasodilatation, such as alcohol, may precipitate an acute pain crisis.

The most common location of lesions are the metaphysis or diaphysis of long bones (75\%), but it can affect the posterior element of the spine (10\%), hands, or feet (12\%). In the hand, the proximal phalanx is the most typically affected site, while lesions of the metacarpals and distal phalanges are rarely encountered. The index finger is the most commonly involved digit, while the thumb is the least affected digit. Radiographically, the lesion presents as a small (typically less than $1 \mathrm{~cm}$ in diameter) lucent lesion (nidus) with extensive surrounding bone sclerosis, usually based within the cortex [5]. Histologically, the nidus is composed of highly vascular, richly interwoven osteoid trabeculae and mineralized matrix with a fibrovascular stroma [8].

There have only been a small number of reported cases of osteoid osteoma of the thumb. This is a case of an 18year-old man who underwent surgery for an osteoid osteoma of the distal phalanx of the thumb.

\section{Case Report}

An 18-year-old right-hand-dominant man presented to our office with an approximately 1-year history of left thumb pain without any history of trauma to the area. The pain had progressively increased in severity and became especially unbearable at night; however, it was relieved with oral ibuprofen. At the time of presentation, he was unable to do even light activities with the affected hand secondary to pain. Physical examination demonstrated moderate swelling and tenderness on the radial aspect of the distal phalanx of the left thumb. There was no erythema or induration. Neurovascular exam of the left upper extremity was normal.

Radiographs of the hand demonstrated a broad zone of sclerosis combined with cortical thinning across the radial and volar aspect of the thumb distal phalanx with a central area of lucency (Fig. 1). There was also soft tissue swelling with 
Figure 1 a $\mathrm{AP}$ and $\mathbf{b}$ lateral preoperative views of the thumb in our patient demonstrate a broad zone of sclerosis across the radial aspect of the thumb distal phalanx with some cortical thinning of the volar and radial aspect. The thumb is also diffusely swollen with inflammatory stranding demonstrated along the radial aspect of the thumb.
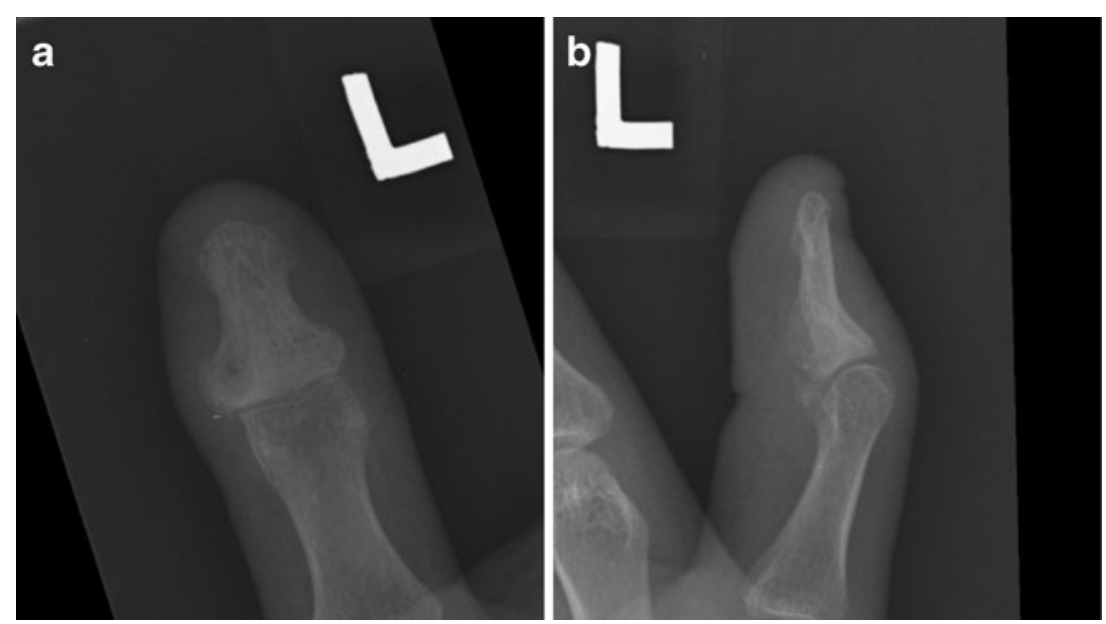

inflammatory stranding seen on the plain films, findings read by the attending radiologist as being consistent with chronic osteomyelitis or cellulitis. The patient also had a magnetic resonance imaging (MRI) study that was also considered consistent with infection. Laboratory examination showed the following: white blood cell count of $8.2 \times 10,000 / \mathrm{mm}^{3}$, rheumatoid factor $=0$, uric acid of $5 \mathrm{mg} / \mathrm{dL}$, erythrocyte sedimentation rate of $6 \mathrm{~mm} / \mathrm{h}$, C-reactive protein of $2 \mathrm{mg} / \mathrm{dL}$, and the patient had negative lyme titers. All of these parameters were within normal limits.

In order to further clarify the diagnosis, computed tomography (CT) scan of the left hand was ordered. This showed a focal lucent lesion at the radial aspect near the base of the distal phalanx. The lesion measured 5 to $6 \mathrm{~mm}$ in diameter, and there was no associated periosteal bone formation. There was a central nidus and surrounding sclerosis in the adjacent bone, the appearance of which is typical of an osteoid osteoma (Fig. 2).

After a thorough discussion with the patient and consideration of his persistent pain and difficulty with taking the NSAIDs, it was decided to proceed with surgery. The lesion was approached through a radial mid-lateral incision and was localized using fluoroscopy. The cortical bone over the lesion was gently burred until the red colored nidus was identified. The nidus was resected with a curette and sent for histopathologic examination (Fig. 3). The high-speed burr was then used to remove an additional 1-2 $\mathrm{mm}$ of surrounding reactive bone to ensure adequate removal of the nidus. Intraoperative frozen culture confirmed that the lesion was benign in nature, and final analysis with permanent slides confirmed it as being an osteoid osteoma with immature benign osteoid in a background of highly vascular connective tissue. At the first postoperative visit, the patient had an obvious decrease in the pain he was experiencing before the surgery. At final follow-up, 6 months after surgery, the patient described no pain and full use of his hand for all activities. Exam showed no swelling or tenderness of the thumb and full range of motion at the interphalangeal and metacarpophalangeal joints. X-ray of the thumb showed resolution of the lesion and replacement with new bone (Fig. 4).

\section{Discussion}

Although osteoid osteoma is a common benign bone lesion, its occurrence in the hand is rare [6]. Therefore, patients with this entity are often presumed to have other disorders more common to the region, delaying accurate diagnosis for an extended period of time. While the patient mentioned above did demonstrate the classic symptoms of osteoid osteoma, namely localized pain that is worse at night and

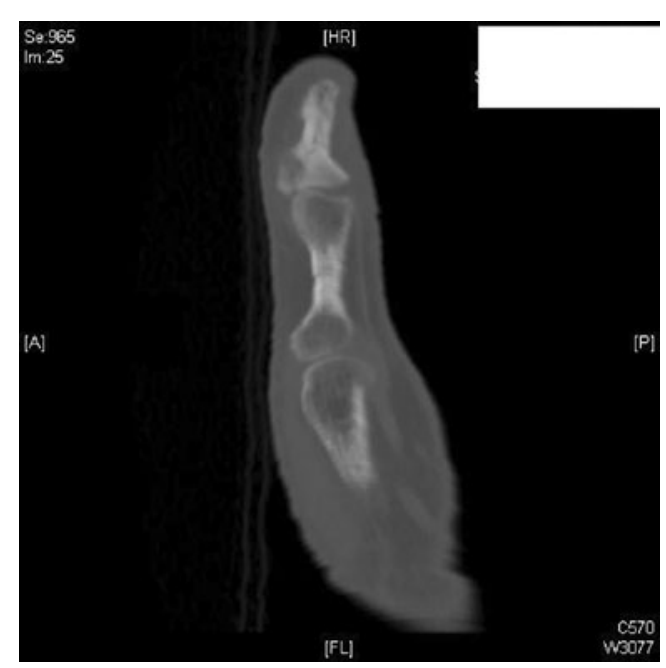

Figure 2 CT scan in the frontal plan demonstrates a focal lytic lesion in the distal phalanx at the radial aspect near the base. The epicenter of this lesion could be periosteal in nature; however, there is no associated periosteal new bone formation. There appears to be a fracture of the lesion through the radial cortex. 


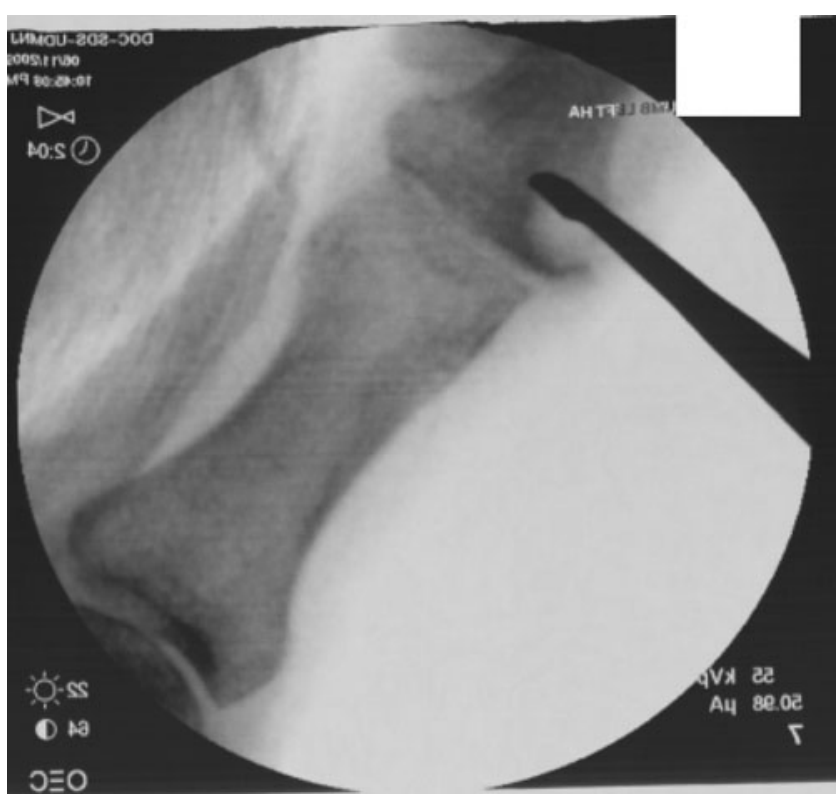

Figure 3 Intraoperative fluoroscopic view localizing the lesion and curetting of its central contents.

relieved by NSAIDs, there have been reports of other patients with this bone lesion in the hand that had atypical symptoms. These include vague, diffuse as opposed to localized pain, fusiform as opposed to localized swelling of the digit, and decreased range of motion with periarticular lesions [9]. Other authors have also reported presentations of profuse digital tip perspiration and intense autonomic phenomena, which may mimic peripheral nerve compression or reflex sympathetic dystrophy [13].

Ramesh et al. reported two cases of 24- and 28-year-old men with lesions in the little and index fingers. The distal phalangeal osteoid osteomas were successfully treated with surgical excision [14]. Di Gennaro et al. in the Italian literature described a case of such a tumor in the distal phalanx of the thumb in a 27 -year-old man. The first radiograph was suggestive of enchondroma, while later computed tomography showed an area of increased density inside the osteolytic area. It was successfully treated with operative curettage [4]. Burger and McCarthy reported two other cases (22- and 34-year-old men with lesions in the fifth and index fingers, respectively) treated with partial amputation of the distal phalanx and surgical excision [2]. Both patients were symptom free 2 years after the surgery. Our case is the youngest case reported of an osteoid osteoma in the distal phalanx. It also reinforces that the best modality for accurate diagnosis is CT scan and that surgical resection and curettage provide an effective means of treatment in these cases.

Osteoid osteomas produce exquisite pain that is disproportionate to their size; there are two postulated reasons for this. Within the nidus, nerve fibers are intimately associated with the blood vessels, and these fibers are larger and more numerous in the reactive zone. An excess of cyclooxygenases and prostoglandins within the lesion leads to vasodilation and also decreased nociceptive threshold of nerve endings resulting in sensations of pain [12]. With the pharmacologic decrease in prostaglandin production, NSAIDs often provide predictable pain relief.

Plain radiographic analysis may demonstrate the typical well-circumscribed lesion with a radiolucent nidus; however, this is only found in $65 \%$ of cases involving the phalanges [1]. This may be due to the small size of the lesion in the phalanx and thus the limited amount of surrounding reactive bone. Lesions may exist in a cortical, subperiosteal, or medullary location; extensive periosteal reaction in cortical lesions may obscure the central nidus and make the diagnosis more difficult [8]. In this patient, the lesion was cortically based with diffuse swelling and inflammatory stranding, which was more consistent with osteomyelitis. Differential diagnosis based on radiographs includes enchondroma, subungueal exostosis, osteochondroma, Brodie's abscess, acute osteomyelitis, and posttraumatic conditions [5].

Technitium bone scans and CT may aid in making the diagnosis when initial radiographs are inconclusive [7].
Figure 4 a $\mathrm{AP}$ and $\mathrm{b}$ lateral views of the thumb at 6 months follow-up demonstrated resolution of the lesion and replacement with new bone. The DIP joint is well aligned with no signs of arthrosis.
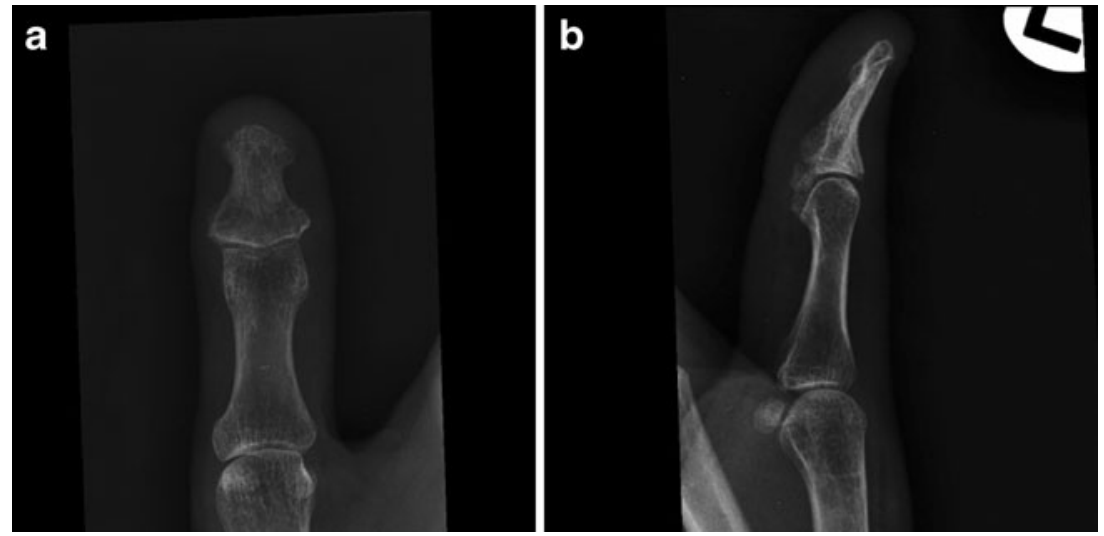
Bone scans are extremely sensitive and will demonstrate significant uptake on immediate and delayed scans secondary to abundant bone formation within the nidus. However, they are not frequently utilized as they have poor specificity in diagnosing an osteoid osteoma. CT scans allow accurate diagnosis and exact localization of the nidus, but fine cuts measuring 1-2 $\mathrm{mm}$ are necessary. It not only has the highest specificity but also allows for assistance with preoperative planning [10]. MRI has been found to be misleading in the diagnosis of an osteoid osteoma, as soft tissue edema surrounding the lesion can produce a falsely aggressive appearance [11]. It might show the lesion, but soft tissue or periosteal edema [2] can make the diagnosis difficult. In our case, MRI was consistent with infection due to soft tissue edema.

Treatment options for patients with osteoid osteoma include nonsurgical management with NSAIDs, CT-guided percutaneous radiofrequency thermal ablation (RFA), and open surgical procedures. Our patient had pain relief with the use of NSAIDs, but developed secondary gastrointestinal intolerance, and therefore the decision was made to proceed with surgical intervention. RFA was considered but was not thought to be appropriate in this location. This technique produces a zone of necrosis $1 \mathrm{~cm}$ in diameter. Its benefit in other locations cannot be disputed as it allows immediate return to full activity and has been shown to have recurrence rates of less than $10 \%$. However, the close proximity of tendons and neurovascular structures in the phalanges to the lesion (and zone of necrosis) puts these structures at risk of injury. In addition, patients may develop joint stiffness secondary to capsular damage [4]. While one case report described a favorable outcome in a patient with osteoid osteoma of the proximal phalanx treated with RFA [15], further studies are necessary to verify its safety, and with the current technology, it is not appropriate for distal phalangeal lesions. Zouari et al. reported the results of CT-guided percutaneous laser photocoagulation in 15 patients with osteoid osteomas of the hands and feet. Only one lesion was in the head of proximal phalanx of third finger. All except one patient were symptom free throughout the follow-up period (24 months). This case was of a 12-year-old girl who had a lesion in the neck of talus. She was treated with a repeat procedure and was symptom free at the last follow-up visit (45 months later) [16].
Osteoid osteoma should always be considered in cases with phalangeal pain especially if the pain is worse at night time and subsides with NSAIDS. Computed tomography not only has the highest specificity but also allows for accurate preoperative planning. Surgical resection, curettage, and additional burring of the surrounding bone is an effective means of treatment in these cases. Recurrence rates of less than $10 \%$ have been reported with this technique [3].

\section{References}

1. Allieu Y, Lussiez B, Benichou M, et al. A double nidus osteoid osteoma in a finger. J Hand Surg Am. 1989;14(2738342):538-41.

2. Burger IM, McCarthy EF. Phalangeal osteoid osteomas in the hand: a diagnostic problem. Clin Orthop Relat Res. 2004;427:198-203.

3. Campanacci M, Ruggieri P, Gasbarrini A, et al. Osteoid osteoma. Direct visual identification and intralesional excision of the nidus with minimal removal of bone. J Bone Jt Surg Br. 1999;81 (10530842):814-20.

4. Di Gennaro GL, Lampasi M, Bosco A, et al. Osteoid osteoma of the distal thumb phalanx: a case report. Chir Organi Mov. 2008;92 (19052928):179-82.

5. Greenspan A. Orthopedic radiology: a practical approach. 2nd ed. New York: Gower Medical; 1992.

6. Grundberg AB. Osteoid osteoma of the thumb: report of a case. J Hand Surg Am. 1977;2(893981):266.

7. Inagaki H, Inoue G. Osteoid osteoma of the distal phalanx. Orthopedics. 1999;22(10580829):1093-4.

8. Johnson T, Steinbach L. Essentials of musculoskeletal imaging. 1st ed. Illinois: American Academy of Orthopedic Surgeons; 2004.

9. Levy Y, Rosenheck S, Greiff M, et al. Osteoid osteoma of the distal phalanx of the thumb. Acta Orthop Scand. 1979;50(6 Pt 1):667-9.

10. McGrath BE, Bush CH, Nelson TE, et al. Evaluation of suspected osteoid osteoma. Clin Orthop Relat Res. 1996;(327):247-52. PMID: 8641070

11. Mirra J, Picci P, Gold R. Bone tumors: clinical, radiologic, and pathologic correlations. 2nd ed. Philadelphia: Lea and Febiger; 1989.

12. Mungo DV, Zhang X, O'Keefe RJ, et al. COX-1 and COX-2 expression in osteoid osteomas. J Orthop Res. 2002;20(1):159-62.

13. Nakatsuchi Y, Sugimoto Y, Nakano M. Osteoid osteoma of the terminal phalanx. J Hand Surg Br. 1984;9(6747431):201-3.

14. Ramesh P, Khan F, Kamboj H. Painful lesions at the fingertips. J R Soc Med. 2004;97(1):30-1.

15. Ramos L, Santos JA, Santos G, et al. Radiofrequency ablation in osteoid osteoma of the finger. J Hand Surg Am. 2005;30 (16039375):798-802.

16. Zouari L, Bousson V, Hamze B, et al. CT-guided percutaneous laser photocoagulation of osteoid osteomas of the hands and feet. Eur Radiol. 2008;18(11):2635-41. 